DOI: 10.1002/adma.201702210

Article type: Communication

\title{
Ferroelectrics as smart mechanical materials
}

Kumara Cordero-Edwards*, Neus Domingo, Amir Abdollahi, Jordi Sort, and Gustau Catalan*

K. Cordero-Edwards, Dr. N. Domingo, Prof. G. Catalan

Institut Català de Nanociencia i Nanotecnologia (ICN2), CSIC and The Barcelona Institute of Science and Technology (BIST), Campus UAB, Bellaterra, 08193 Barcelona, Spain

Email: kumara.cordero@icn2.cat, gustau.catalan@icn2.cat

Dr. A. Abdollahi

Laboratori de Càlcul Numèric (LaCàN), Universitat Politècnica de Catalunya (UPC), Campus Nord UPC-C2, E-08034 Barcelona, Spain

Prof. J. Sort

Departament de Física, Universitat Autònoma de Barcelona (UAB), Edifici Cc, E-0819

Bellaterra, Spain

Prof. J. Sort, Prof. G. Catalan

${ }^{3}$ Institució Catalana de Recerca i Estudis Avançats (ICREA), Pg. Lluís Companys 23, E08010 Barcelona, Spain

Keywords: flexoelectricity, ferroelectricity, mechanical properties, ferroelectric memories, mechanical reading polarity.

The mechanical properties of homogeneous materials are insensitive to space inversion, even when they are crystallographically asymmetric. In practice, this means that turning a piezoelectric upside down or switching the polarization of a ferroelectric should not change its mechanical response. Strain gradients, however, introduce an additional source of asymmetry that has mechanical consequences. Using nanoindentation and contact-resonance force microscopy, we demonstrate that the mechanical response of an uniaxial ferroelectric $\left(\mathrm{LiNbO}_{3}\right)$ does change when its polarity is switched, and use this to quantify its flexoelectricity and to mechanically read the sign of its ferroelectric domains. 


\section{WILEY-VCH}

The mechanical properties of homogeneous materials (stiffness, hardness, toughness and so on) are insensitive to space inversion, because all the magnitudes involved (stress, strain, elastic constants) are described by even parity tensors. This mathematical argument is even valid for cristallographically asymmetric materials such as ferroelectrics and piezoelectrics, and physically, this means that the mechanical response of a ferroelectric material should not depend on whether its polar axis is pointing up or down. However, symmetry restrictions change when deformations are inhomogeneous. ${ }^{[1]-[4]}$ For example, flexoelectricity (coupling between polarization and strain gradient) allows switching ferroelectric polarization by mechanical means, something that would be otherwise symmetry-forbidden if the strain was homogeneous. ${ }^{[5]-[6]}$ Flexoelectricity can also affect the mechanical response. ${ }^{[7]-[9]}$ Importantly, the incorporation of strain gradients (a third-rank tensor) breaks spatial inversion symmetry, so it allows asymmetric mechanical behaviour. In ferroelectrics, polarization also breaks inversion symmetry and may cooperate or compete with flexoelectricity. Thus, by switching ferroelectric polarization one can affect the total polarization, resulting in a material whose mechanical response to strain gradients can be switched with a voltage. By analogy with smart functional materials (materials whose functional response is sensitive to external input fields), ferroelectrics can be viewed as "smart mechanical materials", whose mechanical response to strain gradients can be switched with a voltage.

It is the purpose of this paper to demonstrate that the mechanical response of a ferroelectric is indeed switchable. We show that mechanical asymmetry not only affects toughness, as theoretically predicted, ${ }^{[10]}$ but also all other mechanical properties, both plastic and elastic. This discovery, in turn, enables the use of purely mechanical means to quantify flexoelectricity, or to determine the sign of a ferroelectric domain (or, eventually, a ferroelectric memory bit) by just poking it. Both of these concepts are demonstrated here. 


\section{WILEY-VCH}

Physically, an asymmetric mechanical response can be rationalized by considering the energy cost of deforming a piezoelectric material that generates a polarization $\boldsymbol{P}$ in response to the deformation. This energy cost has two contributions: an elastic one, associated with the deformation itself (Hooke's law), and an electrostatic one, associated with the deformationinduced polarization. The electrostatic energy is $1 / 2 \chi^{-1} \boldsymbol{P}^{2}$, where $\chi$ the dielectric susceptibility is. Because the polarization is squared, the electrostatic energy is insensitive to its sign, so turning a piezoelectric crystal upside-down will not make it any softer. However, when the deformation is inhomogeneous, there are two sources of polarization: the strain itself, via piezoelectricity, and the strain gradient, via flexoelectricity. In a ferroelectric, which is a switchable piezoelectric, these two can be parallel or antiparallel depending on the ferroelectric polarity. Thus, the same inhomogeneous deformation will generate an enhanced polarization when piezoelectricity and flexoelectricity are parallel $\left(\boldsymbol{P}=\boldsymbol{P}_{\text {piezo }}+\boldsymbol{P}_{\text {flexo }}\right)$ and a reduced polarization if they are antiparallel $\left(\boldsymbol{P}=-\boldsymbol{P}_{\text {piezo }}+\boldsymbol{P}_{\text {flexo }}\right)$. The depolarization energy still depends on the square of the total polarization $\left(\boldsymbol{P}^{2}\right)$, but the magnitude of $\boldsymbol{P}$ now depends on the sign of $\boldsymbol{P}_{\text {piezo }}$, so the cost of deformation becomes sensitive to polarity.

It is possible to investigate the mechanical response of ferroelectrics to inhomogeneous strain using nanoindentation, which generates flexoelectricity around a sharp indenter tip (Figure 1(a)). The samples studied are single crystals of Lithium Niobate $\left(\mathrm{LiNbO}_{3}\right)$. We have chosen this material because it is a uniaxial ferroelectric, meaning that only $180^{\circ}$ domain switching is possible. This feature prevents any stress-induced ferroelastic reorientation of the polarization, ${ }^{[11]}$ thus simplifying the analysis; however, the arguments used here can also be applied to thin films of ferroelastic-ferroelectrics such as $\mathrm{BaTiO}_{3}$, $\mathrm{PbTiO}_{3}$ or $\mathrm{BiFeO}_{3}$, provided they are epitaxially constrained to have their polarization out-ofplane. 


\section{WILEY-VCH}

Depending on $\mathrm{Li}^{+}$concentration, $\mathrm{LiNbO}_{3}$ can be stoichiometric or congruent. The latter has defect dipoles that can introduce an extrinsic asymmetry. ${ }^{[12]-[13]}$ Here we have studied samples of both types: stoichiometric and congruent. The stoichiometric sample was single-domain (SLN), so space inversion was achieved by just splitting the crystal in two and turning one half upside-down. The congruent sample was periodically poled (PPLN), so both polarities were accessible on the same side. The results between SLN and PPLN were mutually consistent, indicating that sample stoichiometry or switching method do not affect the outcome.

Indentations were first performed in the monodomain crystal, z-cut (polarization perpendicular to the surface) and split with one half placed with polarization pointing up and the other pointing down. Both sides were equally polished to mirror-like appearance. In order to get statistically meaningful results, we performed and analyzed 50 indentations for each mechanical load (25 for each polarity) and measured applying 4 different maximum loads, i.e., a total of 200 indentations. Another set of 100 indentations (50 for each polarity) at a single load were performed on the PPLN. Further details of the nanoindentation measurements are provided in the Methods section, and the full dataset of raw results is provided in the Supporting Online Material.

Figure 1(b) is a schematic of the load-displacement $(F-h)$ curve for a Berkovich indenter. During the loading process, the material undergoes both elastic and plastic deformation. The total energy related to this process is $U_{T}=\int_{0}^{h_{\max }} F d h$, where $h_{\max }$ is the maximum depth reached during loading and $F$ is the force applied by the indenter. The elastic deformation is recovered upon unloading; therefore, the elastic energy can be measured as $U_{e}=\int_{h_{f}}^{h_{\max }} F d h$, where $h_{f}$ is the final indentation depth after complete unloading. Subtracting the total and elastic energies results in the plastic energy $U_{p}=U_{T}-U_{e}$, see the inset in Figure 1(b). 


\section{WILEY-VCH}

We define asymmetry as $\%$ Asym $\equiv 100 \frac{\left\langle M^{+}\right\rangle-\left\langle M^{-}\right\rangle}{\langle M\rangle}$, where $\left\langle M^{+}\right\rangle-\left\langle M^{-}\right\rangle$is the difference between the mean mechanical indentation energies of the up-polarized and downpolarized states, while $\langle M\rangle$ is the average for all polarities. Positive (negative) asymmetry indicates a larger value for the upward (downward) polarization. Figure 1(d) shows the asymmetry of the elastic, plastic, and total indentation energies as a function of the maximum indentation load. The total energy (elastic + plastic) is symmetric, reflecting that the mechanical energy provided by the indenter is independent of sample polarity, as it should. By contrast, asymmetry is observed for the plastic energy and thus also by the plasticity index (Figure 1S), which is the dimensionless parameter indicating the ratio of plastic energy to total energy, $U_{p} / U_{t}$. Since fracture toughness is proportional to plasticity index ${ }^{[14],[15]}$ crack propagation can be sensitive to the sign of polarization. ${ }^{[9][[10]}$ The elastic energy (Figure 1(d)) is also asymmetric, and this implies that not only plastic but also elastic responses must be polarity-dependent. Using the Oliver-Pharr method, ${ }^{[16],[17]}$ we have quantified one plastic and one elastic response: (a) hardness, as a measure of resistance to plastic deformation, and (b) contact stiffness, as a measure of the elastic response of the material. Both are found to depend on polarity (see Figure S1).

Having demonstrated that flexoelectricity induces mechanical asymmetry, we can use this asymmetry to quantify the flexoelectric coefficient of ferroelectrics. We do this for two reasons (i) to validate quantitatively that the flexoelectric origin of the mechanical asymmetry and (ii) to demonstrate that flexocoupling coefficients can be measured by mechanical means. In piezoelectrics, finding a new and reliable way to measure flexoelectricity is particularly important because the conventional methods (electromechanical rather than mechanical) yield unrealistically high results ${ }^{[3]}$ due to piezoelectric contributions. ${ }^{[18],}{ }^{[19]}$ We have derived a simplified analytical expression (see Supporting Online Material) relating the flexocoupling coefficient to the difference in free energy $(\Delta G)$ between the up and down polarized states: 


\section{WILEY-VCH}

$$
f=\frac{1}{6} \frac{\bar{E} \Delta G}{P_{0} F}
$$

where $P_{0}$ is the ferroelectric spontaneous polarization $\left(0.8 \mathrm{C} / \mathrm{m}^{2}\right.$ for $\left.\mathrm{LiNbO}_{3}\right),{ }^{[12]} F$ is the maximum indentation load and $\bar{E}$ is the average of the elastic modulus measured for the upand down-polarized states. Experimentally, the energy difference $\Delta G$ can be obtained by subtracting the measured elastic energy (area under the unloading curve in Figure 1(b)) of the upward and downward polar states, i.e. $\Delta G=U_{e}^{+}-U_{e}^{-}$.Using the values obtained experimentally at $7 \mathrm{mN}$, and Equation (1) the resulting flexocoupling coefficient $f$ of SLN is $54 \pm 4 \mathrm{~V}$, and for PPLN is $40 \pm 5 \mathrm{~V}$.

The obtained flexocoupling coefficients are still somewhat larger than the KoganTagantsev expectation value $f<10 \mathrm{~V},,^{[1]-[3]}$ but the order-of-magnitude agreement is nevertheless remarkable considering the simplifications made in order to obtain the analytical expression in eq. (1) (see Supporting Online Material). The accuracy also represents an enormous improvement compared to beam-bending experiments, which for ferroelectrics always yield flexocoupling coefficients that are many orders of magnitude too large. ${ }^{[3]}$

Another notable consequence of these results is that they allow determining the polarity of a ferroelectric just by indenting its surface. This is demonstrated by contact stiffness measurements performed on PPLN (Figure 2), which show that downward polarized material is stiffer while the upward-oriented one is more flexible.

Nanoindentation is, by definition, a destructive method, but since stiffness is an elastic property it is not necessary to punch holes in order to read polarity. To prove this point, we use Contact Resonance Force Microscopy $(\mathrm{CRFM})^{[20]}$ (see Methods): with this technique, we deliver an oscillatory force by vibrating the AFM in hard contact with the surface of the ferroelectric. The vibration amplitude peaks when the vibration frequency delivered by the cantilever coincides with the mechanical resonance frequency of the tip-sample contact. 


\section{WILEY-VCH}

Since contact resonance frequency depends on tip-sample mechanical coupling, it is sensitive to the material's stiffness, with higher resonance frequency corresponding to higher stiffness. The sensitivity has been used in the past to evidence contrast in Young's Modulus between domains of different ferroelastic orientation, ${ }^{[21]-[22]}$ but the technique was thought to be blind with respect to polarization sign. Our results, however, show that there is a measurable difference between the contact resonance frequency of oppositely-polarized domains (Figure 3), with down-polarized domains resonating at higher frequencies (stiffer) than up-polarized ones, in agreement with the nanoindentation results. This result is explained by the same arguments as in the indentation experiment: inhomogeneous deformation under the AFM tip induces a flexoelectric polarization that either adds to or subtracts from the piezoelectricity of the domains depending on their ferroelectric sign, resulting in asymmetric energy costs of deformation and thus different stiffness and contact resonance frequency. These results thus evidence the importance of tip-induced gradient effects for tip-sample coupling in AFM, and demonstrate that such gradient effects can be exploited to mechanically read polarity.

To summarize: the results indicate that the mechanical responses (plastic and elastic) of a ferroelectric to inhomogeneous deformation are asymmetric and switchable. This switchable asymmetry was used both to quantify the flexoelectric coefficient itself and to determine the polar sign of a ferroelectric domain by purely mechanical means. This demonstration opens up new device possibilities such reading a ferroelectric memory without electrodes, or making coatings whose mechanical response to localized deformations (e.g. scratching) can be modified with a voltage. Nowadays ferroelectrics are already considered as smart multifunctional materials on account their switchable polarization and electromechanical response. Our results show that they can also be considered as smart mechanical materials. 


\section{WILEY-VCH}

\section{Experimental Section}

Sample preparation and characterization: Depending on the growth process, single crystals can be stoichiometric, with a ratio of $1: 1: 3$ for $\mathrm{Li}^{+}: \mathrm{Nb}^{+5}: \mathrm{O}^{-2}$, or (the most common) congruent which exhibit $\mathrm{Li}^{+}$deficiency. Such lithium vacancies can result in defect dipoles that may be either parallel or antiparallel to the ferroelectric polarization, thus introducing an additional and extrinsic source of asymmetry ${ }^{[12]-[13]}$ that can complicate the analysis of the results. To guarantee that any evidence of asymmetry originates from flexoelectricity, we use Stoichiometric Lithium Niobate (SLN) single crystals, purchased from MTI Corporation. These are single-domain, so the SLN z-cut single crystal was cut in two equal pieces and we turned one upside-down in order to study two areas with opposite polarization. Both crystals were chemical cleaned. They were sonicated for $15 \mathrm{~min}$ in acetone, isopropanol and MilliQ water sequentially. Finally, both were glued in a metallic disc with silver paste, one with the polarization pointing upward and the other downward. We also studied a periodically-poled lithium niobate (PPLN) crystal provided by Asylum Research, Santa Barbara, CA., and chemically cleaned like the SLN. These crystals were congruent; there are no commercially available stoichiometric crystals of PPLN. The polarization of the samples was checked by Piezoresponse Force Microscopy (PFM) using an MFP-3D AFM from Asylum Research, Santa Barbara, CA.

Nanoindentation: Experiments were carried out in the load-control mode, using a UMIS instrument from Fischer-Cripps Laboratories equipped with a Berkovich pyramidal-shaped diamond tip. The thermal drift was always kept below $\pm 0.05 \mathrm{nms}^{-1}$. Four different loads (7 $\mathrm{mN}, 10 \mathrm{mN}, 15 \mathrm{mN}$ and $20 \mathrm{mN}$ ) were applied. To ensure statistical robustness and accuracy of the results a total of 50 indents per load (25 in each polar state, see Figure 1c)) were performed in the SLN single crystal, and a total of 100 indents per load in the PPLN single 


\section{WILEY-VCH}

crystal. Indents were spaced $15 \mu \mathrm{m}$ apart (see Figure 1c)), ensuring a sufficient independence of the indents in all cases.

PFM images on PPLN: To correlate the direction of the polarization with each indentation, PFM experiments were carried out, using an MFP-3D AFM, and OMCL - AC240TM - R3 cantilevers, with a $\mathrm{k} \sim 2 \mathrm{~N} / \mathrm{m}$. PFM was mainly operated in DART mode to benefit from resonance signal enhancement; in PFM, an electrical $a c$ signal is applied to the tip used as top electrode that excites the sample and mechanical response due to inverse piezoelectric effect is monitored.

CRFM images on PPLN: Experiments were carried out using an MFP-3D AFM, in a controlled ambient with $\mathrm{N}_{2}$. In CRFM a mechanical ac excitation signal is applied to the cantilever in contact with the surface, and the resonance frequency is monitored, in this case also operating in DART mode. The mechanical resonance of the cantilever in contact with the surface strongly depends on the coupling with the mechanical properties of the surface. Nanosensors NCL Pt coated tips, with $\mathrm{k} \sim 48 \mathrm{~N} / \mathrm{m}$ were used. The contact force between the cantilever and the sample was about $25 \mu \mathrm{N}$.

\section{Supporting Information}

Supporting Information is available from the Wiley Online Library or from the author.

\section{Acknowledgements}

K. C-E and G.C. acknowledges ERC Starting Grant 308023. Financial support has been obtained under projects from the Spanish Ministerio de Economía y Competitividad (MINECO) under projects FIS2013-48668-C2-1-P and FIS2015-73932-JIN, and the MAT2014-57960-C3-1-R cofinanced by the 'Fondo Europeo de Desarrollo Regional' (FEDER). ICN2 acknowledges support from the Severo Ochoa Program (MINECO, Grant SEV-2013-0295). This work has also been partially funded by 2014-SGR-1015 and 2014SGR-1216 projects from the Generalitat de Catalunya.

Received: ((will be filled in by the editorial staff))

Revised: ((will be filled in by the editorial staff)) Published online: ((will be filled in by the editorial staff)) 


\section{WILEY-VCH}

References

[1] S. Kogan, Sov. Phys. Solid State 1964, 5, 2069-2079.

[2] A. K. Tagantsev. Phys. Rev. B 1986, 34, 5883.

[3] P. Zubko, G. Catalán, A.K. Tagantsev. Annu. Rev. Mater. Res. 2013, 43: 387 - 421.

[4] R. S. Lakes, R.L. Benedict. Int. J. Engng Sci. 1982, 29, 1161.

[5] H. Lu, C. -W. Bark, D. Esque de los Ojos, J. Alcalá, C. B. Eom, G. Catalán, A. Gruverman. Science 2012, 336, 59.

[6] J. Ocenásek, H. Lu, C. W. Bark, C. B Eom, J. Alcalá, G. Catalan, A. Gruverman. Phys. Rev B. 2015, 92, 035417.

[7] M. Gharbi, Z.H. Sun, P. Sharma, K. White. Appl. Phys. Lett. 2009, 95, 142901.

[8] C.R. Robinson, K.W. White, P. Sharma. Appl. Phys. Lett 2012, 101, 122901.

[9] H. Zhou, Y. Pei, F. Li, H. Luo, D. Fang. Appl. Phys. Lett 2014, 104, 061904.

[10]A. Abdollahi, C. Peco, D. Millán, M. Arroyo, G. Catalán, I. Arias. Phys. Rev B 2015, 92, 094101.

[11]M.J. Reece, F. Guiu. Philosophical Magazine A 2002, 82, 1: 29-38.

[12] S. Kim, V. Gopalan, K. Kitamura, Y. Furukawa. Y. J. Appl. Phys 2001, 90, 2949.

[13] V. Gopalan, V. Dierolf, D.A. Scrymgeour. Annu. Rev. Mater. Res 2007, 37, 449 - 489.

[14] G.M. Pharr. Mater. Sci. Eng. A 1998, 253, 151-159.

[15] Y. -T- Cheng, C. -M. Cheng. Appl. Phys. Lett. 1998, 73, 614-616.

[16] W.C. Oliver, G.M. Pharr. J. Mater. Res. 1992, 7, 1564 - 1583.

[17]Fisher - Cripps, A.C. Nanoindentation. 2002, $3^{\text {rd }}$ Ed. Springer-Verlag, New York.

[18] J. Narvaez, S. Saremi, J. Hong, M. Stengel, G. Catalan. Phys. Rev. Lett. 2015, 115, 037601.

[19] A. Biancoli, C.M. Fancher, J.L. Jones, D. Damjanovic, D. Nat. Mat. 2015, 14, 224-229 .

[20] U. Rabe, S. Amelio, E. Kester, V. Scherer, S. Hirsekorn, W. Arnold, Ultrasonics 2000, 38, 430-437.

[21] U. Rabe, M. Kopycinska, S. Hirsekorn, J. Muñoz-Saldaña, G.A. Schneider, W. Arnold, J. Phys. D: Appl. Phys. 2002, 35, 2621-2635.

[22] X. L. Zhou, F.X. Li, H.R Zeng, H. R. J. Appl. Phys. 2013, 113, 187214. 
WILEY-VCH
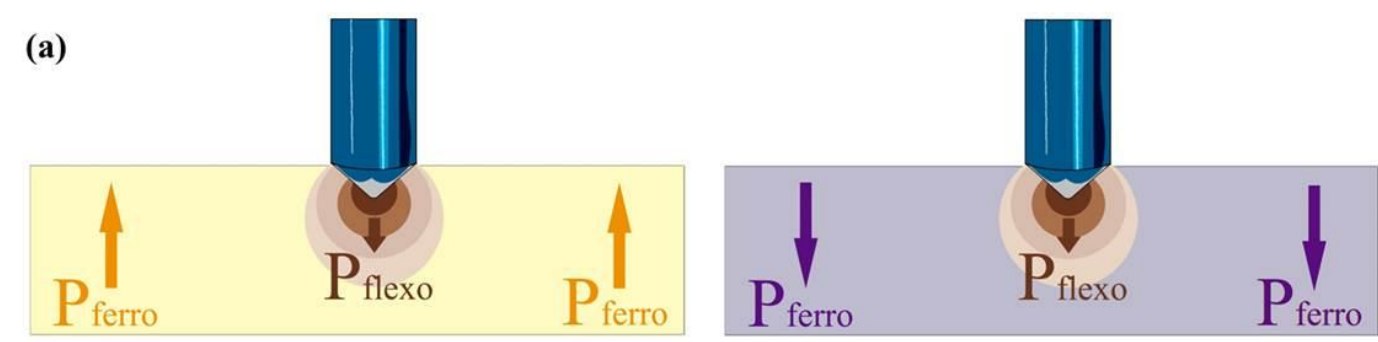

(b)

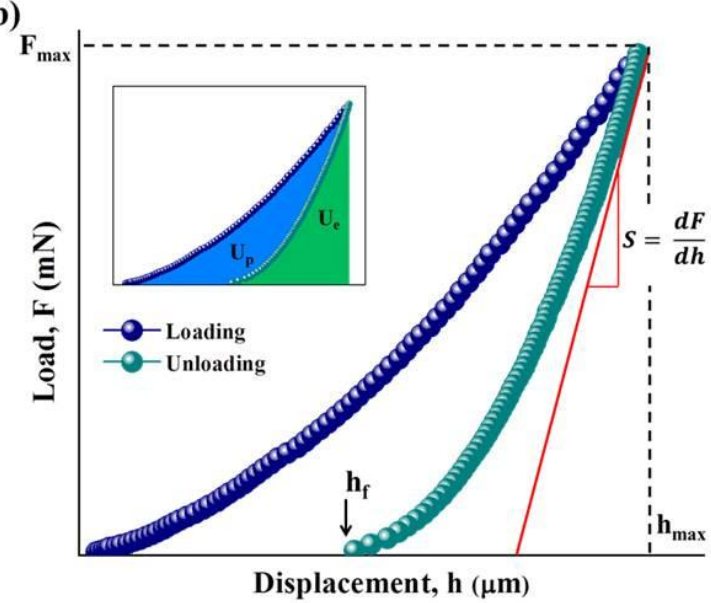

(c)

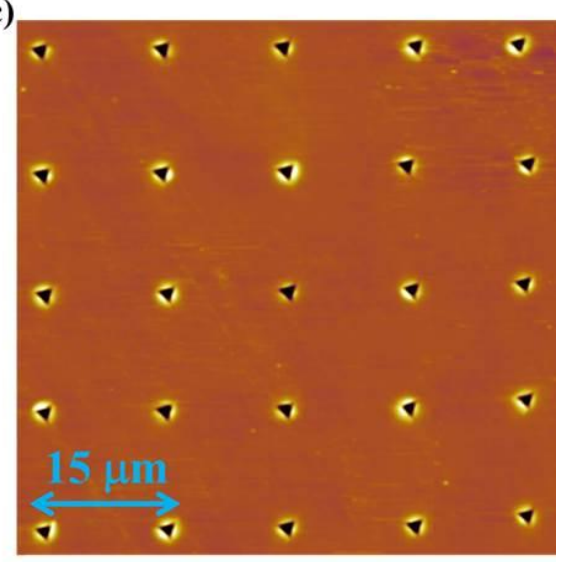

(d)

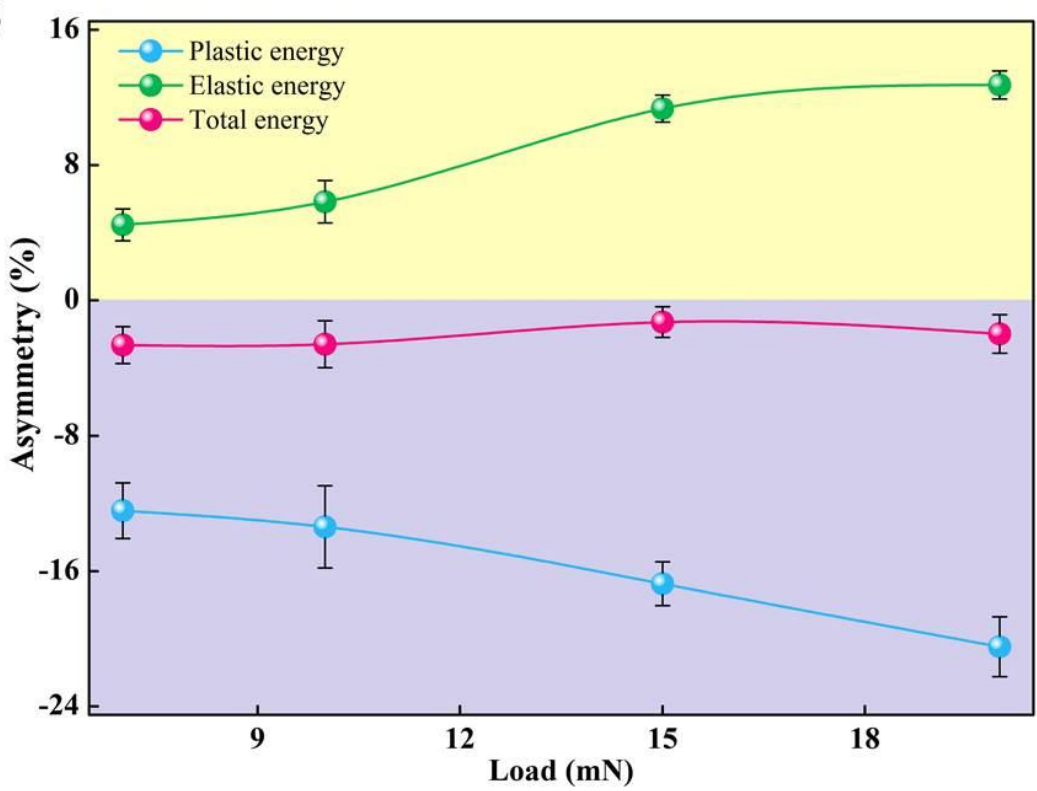

Figure 1: (a) Schematic of the strain gradient field and the associated polarizations (arrows) induced by the indenter tip on a uniaxial ferroelectric with polarization pointing up (left), and polarization pointing down (right). (b) Schematic of the loading and unloading forcedisplacement curve performed by the nanoindenter, from which the energies (inset), and mechanical parameters are obtained. (c) AFM topography image of the surface of an SLN crystal after performing 25 nanoindentations with the same indentation force. (d) Asymmetry behavior of energies as a function of the maximum indentation load in SLN single crystals, showing that both plastic and elastic mechanical responses are asymmetric. 


\section{WILEY-VCH}

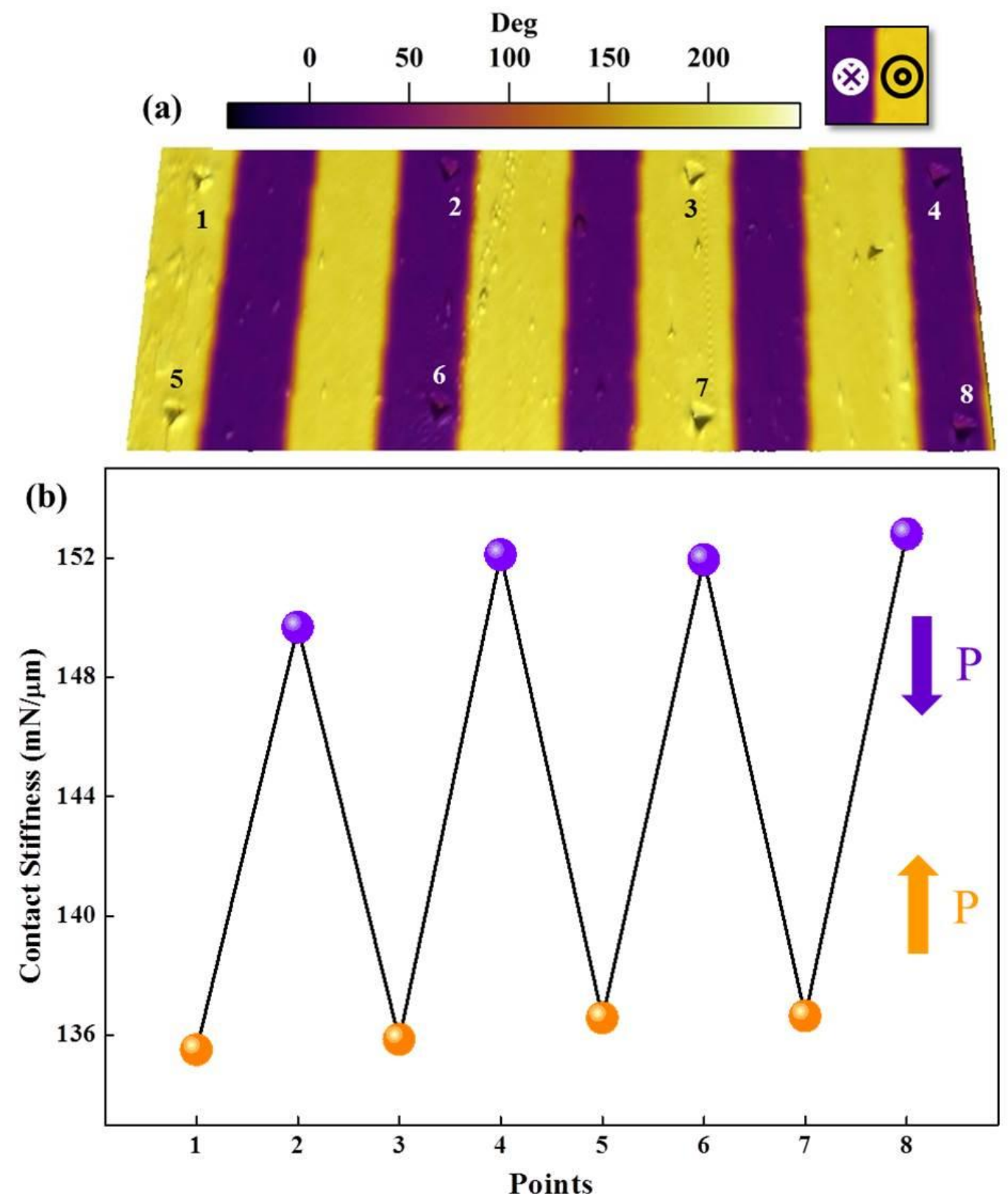

Figure 2: a) 3D plot of topography with superimposed PFM phase image of a few indents performed in PPLN at $7 \mathrm{mN}$. Yellow means that the polarization is pointing up, whereas purple means that it is pointing down. b) Contact Stiffness measured as a function of the number of indent in (a), showing that the relative stiffness is a direct indicator of a polar state, and therefore it is possible to "read" the polarization of a ferroelectric from its mechanical response. 


\section{WILEY-VCH}

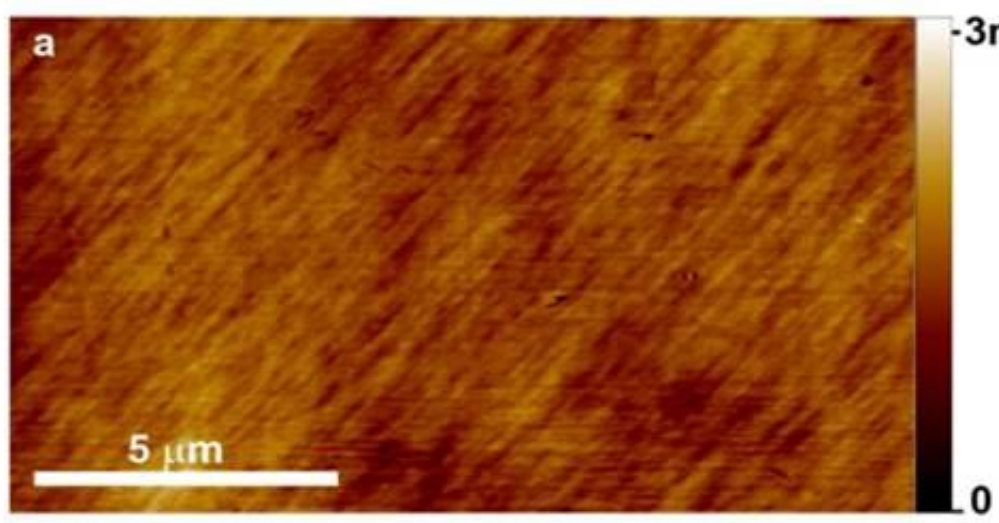

$-3 \mathrm{~nm}$
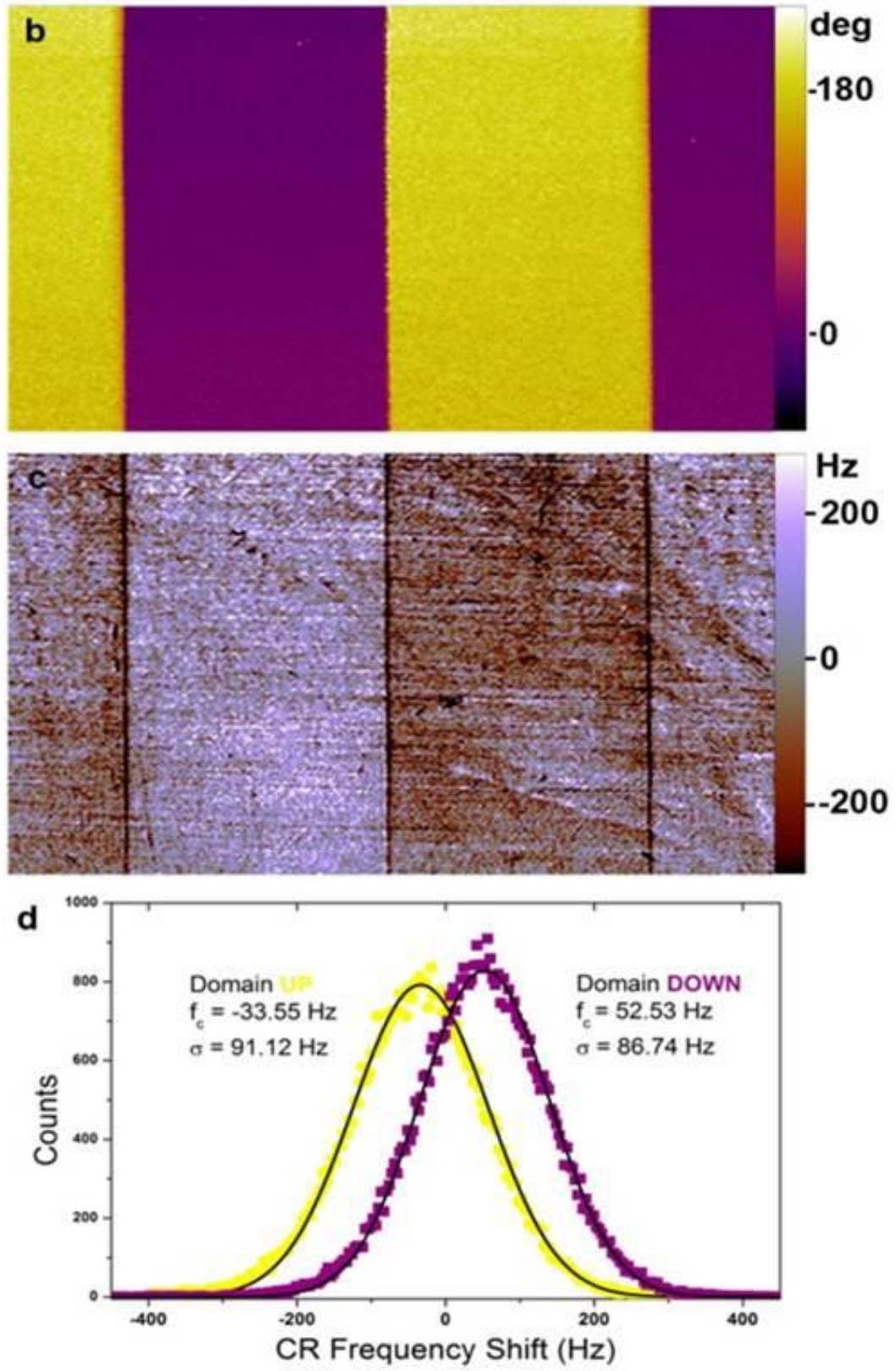

Figure 3: a. Topography and b. Phase PFM image showing the polarization of the domains in a periodically pooled $\mathrm{LiNbO}_{3}$ sample (PPLN), with $\mathrm{Ph}_{\mathrm{PFM}}=0^{\circ}$ for domains pointing down and $\mathrm{Ph}_{\mathrm{PFM}}=180^{\circ}$ for domains pointing up. c. Contact resonance frequency mapping of the PPLN surface, the contact resonance frequency is shifted towards higher frequencies for down-polarized domains, meaning they are stiffer, and to lower frequencies for the up- 


\section{WILEY-VCH}

polarized domains, meaning they are softer. $\mathbf{d}$. Histogram of the CR-AFM image shown in c: the yellow dots correspond to the frequency shift counts in the areas associated to domains pointing up and purple squares to domains pointing down. Black lines are the corresponding Gaussian fittings, with parameters shown in the inset. The total CR frequency contrast among different polarized domains is of about $\Delta \mathrm{f} \sim 86 \mathrm{~Hz}$, using a cantilever of $\mathrm{k} \sim 48 \mathrm{~N} / \mathrm{m}$. 


\section{WILEY-VCH}

\section{The table of contents}

Ferroelectrics are smart multifunctional materials: their polarization responds to external stimuli (stress, voltage, temperature) for transductors, and can be switched for memories. Here, we show that, thanks to flexoelectricity, their mechanical response to strain gradients is switchable, so that they may also be regarded as mechanically smart. Two practical consequences, demonstrated here, are mechanical measurement of flexoelectricity and mechanical reading of ferroelectric sign.

Keyword mechanical reading polarity

K. Cordero-Edwards*, N. Domingo, A. Abdollahi, J. Sort, and G. Catalan*

Ferroelectrics as smart mechanical materials

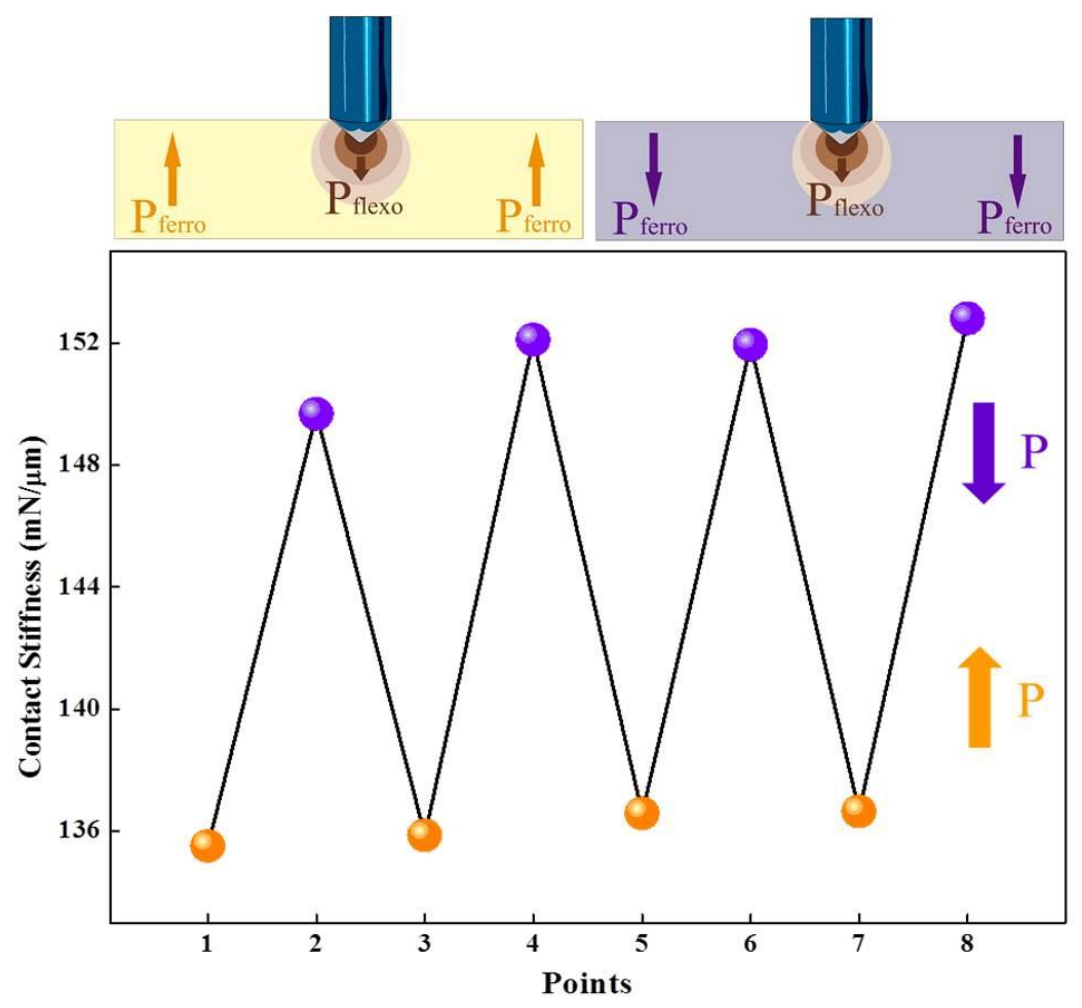




\section{WILEY-VCH}

Copyright WILEY-VCH Verlag GmbH \& Co. KGaA, 69469 Weinheim, Germany, 2016.

\section{Supporting Information}

\section{Ferroelectrics as smart mechanical materials}

Kumara Cordero-Edwards*, Neus Domingo, Amir Abdollahi, Jordi Sort, and Gustau Catalan*

\section{Asymmetry behavior}

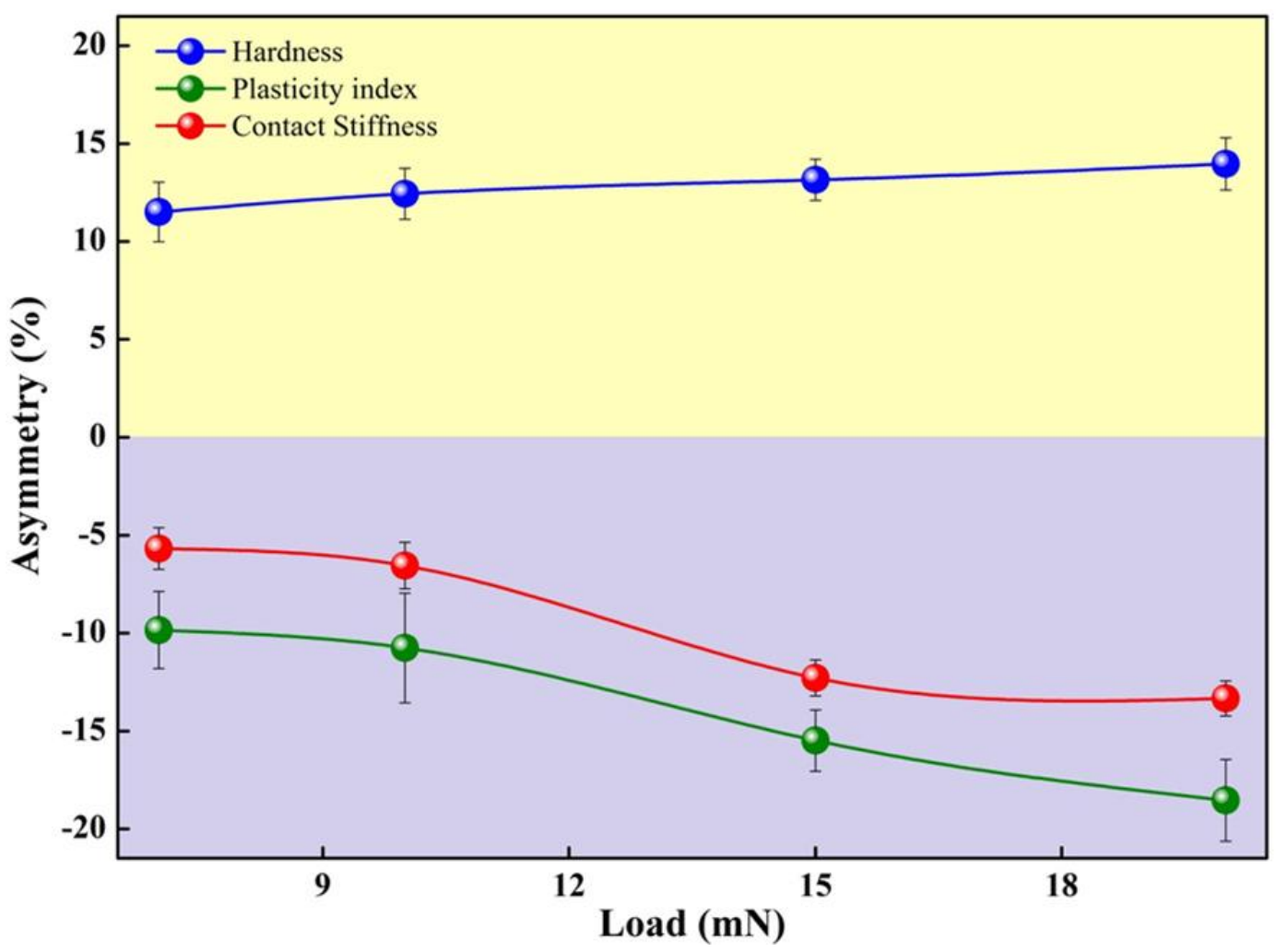

Figure S1: Asymmetry behavior of mechanical properties as a function of the maximum indentation load in SLN single crystals.

\section{Nanomechanics: measuring flexoelectricity from nanoindentation}

The role of flexoelectricity on the response of ferroelectrics can be exposed by their free energy [[9]-[9]]:

$$
\begin{aligned}
& G=\int\left[\frac{1}{2} \alpha_{i j} P_{i} P_{j}+\frac{1}{4} \beta_{i j k l} P_{i} P_{j} P_{k} P_{l}+\frac{1}{6} \gamma_{i j k l m n} P_{i} P_{j} P_{k} P_{l} P_{m} P_{n}+\frac{1}{2} c_{i j k l} \varepsilon_{i j} \varepsilon_{k l}-\frac{1}{2} q_{i j k l} \varepsilon_{i j} P_{k} P_{l}+\right. \\
& 12 \text { gijklPi,jPk,l- fijklPkdcijdxldV, }
\end{aligned}
$$




\section{WILEY-VCH}

where the first three terms represent the Landau free energy density with the phenomenological Landau-Devonshire coefficients [[3]], $P_{i}$ is the polarization, and $\varepsilon_{i j}$ is the strain. The fourth term denotes the elastic energy density of the system and $c_{i j k l}$ is the elastic tensor. The fifth term indicates the coupling between the polarization and strain, where $q_{i j k l}$ is the electrostrictive tensor. The sixth term is the polarization gradient energy density and $g_{i j k l}$ is the correlation energy tensor.

The last term in Equation 1 is the flexoelectric coupling energy density, and $f_{i j k l}$ is the flexocoupling tensor describing both direct and converse flexoelectric effects [[3], [5]]. We can use this equation to calculate the energy difference between the positively $\left(P^{+}\right)$and negatively $\left(P^{-}\right)$polarized states in the presence of a strain gradient. All terms with even powers of polarization remain identical under polar inversion and therefore disappear from the subtraction, leaving the free energy difference as:

$$
\Delta G=G^{+}-G^{-}=\int\left[f_{i j k l} P_{k}^{-} \frac{\partial \varepsilon_{i j}}{\partial x_{l}}-f_{i j k l} P_{k}^{+} \frac{\partial \varepsilon_{i j}}{\partial x_{l}}\right] d V .
$$

Equation 1 and 2 show explicitly that flexoelectricity introduces a difference (bias) into the otherwise symmetric ferroelectric double well.

Considering that $P_{k}^{+}=-P_{k}^{-} \equiv P_{k}$, Equation 2 can be simplified to:

$$
\Delta G=-2 \int f_{i j k l} P_{k} \frac{\partial \varepsilon_{i j}}{\partial x_{l}} d V
$$

Starting from Equation 3, and considering an isotropic medium, the non-zero independent flexoelectric coefficients $f_{i j k l}$ reduce to two which are the longitudinal $f_{11}$ and transversal $f_{12}$ flexoelectric coefficients [[6]]. By assuming that $f_{11}=f_{12}=f$, we can expand the right-hand side of Equation 3 as

$$
\Delta G=-2 \int \begin{gathered}
f\left(P_{1} \frac{\partial \varepsilon_{11}}{\partial x_{1}}+P_{2} \frac{\partial \varepsilon_{22}}{\partial x_{2}}+P_{3} \frac{\partial \varepsilon_{33}}{\partial x_{3}}\right) \\
+f\left(P_{1} \frac{\partial \varepsilon_{22}}{\partial x_{1}}+P_{1} \frac{\partial \varepsilon_{33}}{\partial x_{1}}+P_{2} \frac{\partial \varepsilon_{11}}{\partial x_{2}}+P_{2} \frac{\partial \varepsilon_{33}}{\partial x_{2}}+P_{3} \frac{\partial \varepsilon_{11}}{\partial x_{3}}+P_{3} \frac{\partial \varepsilon_{22}}{\partial x_{3}}\right) .
\end{gathered}
$$


Considering $x_{3}$ as the uniaxial direction of the polarization, i.e. $P_{1}=P_{2}=0$, and using Hook's law, $\varepsilon=\sigma / E$, Equation (4) converts to

$$
\Delta G=-\frac{2 f P_{0}}{E} \int\left(\frac{\partial \sigma_{11}}{\partial x_{3}}+\frac{\partial \sigma_{22}}{\partial x_{3}}+\frac{\partial \sigma_{33}}{\partial x_{3}}\right) d V,
$$

where $P_{0}$ is the spontaneous polarization of $\mathrm{LiNbO}_{3}$.

Following an idealized model of indentation [[7]], the surface of the indenter is assumed to be encased in a semi-cylindrical or hemispherical core where there is a hydrostatic stress. The hydrostatic stress can thus be written as $\hat{\sigma}=\left(\sigma_{11}+\sigma_{22}+\sigma_{33}\right) / 3$, and the volume integral can be reduced over the surface as

$$
\Delta G=-\frac{6 f P_{0}}{E} \iint \hat{\sigma} d x_{1} d x_{2}
$$

The stress is $\hat{\sigma}=-F / A$, with $F$ being the indentation force and $A$ the projected area of the elastic contact; Equation 6 therefore can be written as

$$
\Delta G=\frac{6 f F P_{0}}{E}
$$

And thus, the flexocoupling coefficient $f$ is obtained as

$$
f=\frac{1}{6} \frac{E \Delta G}{P_{0} F}
$$

where $P_{0}$ is the spontaneous polarization, $F$ is the maximum indentation load and $\bar{E}$ is the average of the elastic modulus measured for the up- and down-polarized states. 
3. Full dataset of the raw results.

Table 2S1: Data of SLN at $20 \mathrm{mN}$.

\begin{tabular}{|c|c|c|c|c|c|c|c|c|c|c|}
\hline Polarization & Upward & Upward & Upward & Upward & Upward & Downward & Downward & Downward & Downward & Downward \\
\hline Property & Hardness & $\begin{array}{l}\text { Contact } \\
\text { Stiffness }\end{array}$ & $\begin{array}{l}\text { Plastic } \\
\text { energy }\end{array}$ & $\begin{array}{l}\text { Elastic } \\
\text { energy }\end{array}$ & $\begin{array}{l}\text { Total } \\
\text { energy }\end{array}$ & Hardness & $\begin{array}{l}\text { Contact } \\
\text { Stiffness }\end{array}$ & $\begin{array}{l}\text { Plastic } \\
\text { energy }\end{array}$ & $\begin{array}{l}\text { Elastic } \\
\text { energy }\end{array}$ & $\begin{array}{c}\text { Total } \\
\text { energy }\end{array}$ \\
\hline Units & GPa & $\mathrm{m} \mathbf{N} / \mu \mathrm{m}$ & $\mathbf{n J}$ & nJ & $\mathbf{n J}$ & GPa & $\mathrm{mN} / \mu \mathrm{m}$ & $\mathbf{n J}$ & nJ & nJ \\
\hline 1 & 11,752 & 192,056 & 1,006 & 1,440 & 2,446 & 10,341 & 226,202 & 1,192 & 1,250 & 2,443 \\
\hline 2 & 11,403 & 194,497 & 1,036 & 1,435 & 2,470 & 9,678 & 225,350 & 1,223 & 1,283 & 2,507 \\
\hline 3 & 13,543 & 172,647 & 0,777 & 1,633 & 2,411 & 10,087 & 210,315 & 1,196 & 1,305 & 2,501 \\
\hline 4 & 10,475 & 198,356 & 1,147 & 1,405 & 2,552 & 9,915 & 222,163 & 1,214 & 1,271 & 2,485 \\
\hline 5 & 12,151 & 188,545 & 0,959 & 1,471 & 2,430 & 10,094 & 218,708 & 1,172 & 1,269 & 2,441 \\
\hline 6 & 12,465 & 178,021 & 0,903 & 1,542 & 2,445 & 10,096 & 217,117 & 1,204 & 1,302 & 2,505 \\
\hline 7 & 11,161 & 195,336 & 1,019 & 1,444 & 2,462 & 9,823 & 223,407 & 1,249 & 1,267 & 2,516 \\
\hline 8 & 11,629 & 196,885 & 0,961 & 1,454 & 2,415 & 10,291 & 216,104 & 1,185 & 1,295 & 2,481 \\
\hline 9 & 11,584 & 189,569 & 0,951 & 1,480 & 2,431 & 9,870 & 225,795 & 1,240 & 1,268 & 2,508 \\
\hline 10 & 10,726 & 189,684 & 1,106 & 1,462 & 2,568 & 9,928 & 221,348 & 1,272 & 1,272 & 2,544 \\
\hline 11 & 10,568 & 203,513 & 1,067 & 1,409 & 2,476 & 10,864 & 210,678 & 1,089 & 1,335 & 2,424 \\
\hline 12 & 11,624 & 188,125 & 0,967 & 1,484 & 2,451 & 10,137 & 216,430 & 1,211 & 1,299 & 2,510 \\
\hline 13 & 11,200 & 196,563 & 1,049 & 1,437 & 2,486 & 9,874 & 227,553 & 1,244 & 1,250 & 2,494 \\
\hline 14 & 11,464 & 193,235 & 1,026 & 1,415 & 2,441 & 9,782 & 225,216 & 1,236 & 1,263 & 2,499 \\
\hline 15 & 11,389 & 195,052 & 0,995 & 1,435 & 2,430 & 10,182 & 218,732 & 1,187 & 1,282 & 2,468 \\
\hline 16 & 11,859 & 194,569 & 0,907 & 1,478 & 2,386 & 10,180 & 219,187 & 1,235 & 1,293 & 2,529 \\
\hline 17 & 11,850 & 192,679 & 0,956 & 1,452 & 2,408 & 10,079 & 224,258 & 1,180 & 1,275 & 2,455 \\
\hline 18 & 12,001 & 189,463 & 0,911 & 1,481 & 2,392 & 9,485 & 230,833 & 1,325 & 1,229 & 2,554 \\
\hline 19 & 11,129 & 197,929 & 1,046 & 1,435 & 2,482 & 10,540 & 209,041 & 1,139 & 1,316 & 2,455 \\
\hline 20 & 11,601 & 201,389 & 0,968 & 1,441 & 2,409 & 10,235 & 219,079 & 1,207 & 1,277 & 2,484 \\
\hline 21 & 12,166 & 189,412 & 0,890 & 1,472 & 2,361 & 9,342 & 233,409 & 1,318 & 1,235 & 2,553 \\
\hline 22 & 11,525 & 191,415 & 0,968 & 1,445 & 2,413 & 9,651 & 218,668 & 1,268 & 1,294 & 2,562 \\
\hline 23 & 11,355 & 200,279 & 1,024 & 1,398 & 2,423 & 10,535 & 213,393 & 1,126 & 1,337 & 2,463 \\
\hline 24 & 12,034 & 190,025 & 0,909 & 1,501 & 2,410 & 10,954 & 209,371 & 1,073 & 1,373 & 2,445 \\
\hline 25 & 11,508 & 194,437 & 0,987 & 1,463 & 2,449 & 10,321 & 218,615 & 1,146 & 1,302 & 2,448 \\
\hline
\end{tabular}

Table 2S2: Data of SLN at $15 \mathrm{mN}$.

\begin{tabular}{|c|c|c|c|c|c|c|c|c|c|c|}
\hline Polarization & Upward & Upward & Upward & Upward & Upward & Downward & Downward & Downward & Downward & Downward \\
\hline Property & Hardness & $\begin{array}{l}\text { Contact } \\
\text { Stiffness }\end{array}$ & $\begin{array}{l}\text { Plastic } \\
\text { energy }\end{array}$ & $\begin{array}{r}\text { Elastic } \\
\text { energy }\end{array}$ & $\begin{array}{l}\text { Total } \\
\text { energy }\end{array}$ & Hardness & $\begin{array}{l}\text { Contact } \\
\text { Stiffness }\end{array}$ & $\begin{array}{l}\text { Plastic } \\
\text { energy }\end{array}$ & $\begin{array}{l}\text { Elastic } \\
\text { energy }\end{array}$ & $\begin{array}{c}\text { Total } \\
\text { energy }\end{array}$ \\
\hline Units & GPa & $\mathrm{mN} / \mu \mathrm{m}$ & nJ & nJ & nJ & GPa & $\mathrm{mN} / \mu \mathrm{m}$ & nJ & nJ & nJ \\
\hline 1 & 12,001 & 163,999 & 0,663 & 0,924 & 1,587 & 10,600 & 194,336 & 0,736 & 0,801 & 1,537 \\
\hline 2 & 11,385 & 162,409 & 0,660 & 0,952 & 1,611 & 10,196 & 197,152 & 0,753 & 0,808 & 1,560 \\
\hline 3 & 11,778 & 173,440 & 0,670 & 0,888 & 1,559 & 9,855 & 188,069 & 0,776 & 0,878 & 1,653 \\
\hline 4 & 12,102 & 169,698 & 0,621 & 0,914 & 1,535 & 10,206 & 192,441 & 0,753 & 0,810 & 1,564 \\
\hline 5 & 12,506 & 162,703 & 0,596 & 0,945 & 1,542 & 10,000 & 199,278 & 0,813 & 0,782 & 1,595 \\
\hline 6 & 10,934 & 176,849 & 0,713 & 0,892 & 1,606 & 9,641 & 209,635 & 0,828 & 0,786 & 1,613 \\
\hline 7 & 12,376 & 167,828 & 0,590 & 0,945 & 1,534 & 10,934 & 185,795 & 0,696 & 0,853 & 1,550 \\
\hline 8 & 11,179 & 177,457 & 0,675 & 0,895 & 1,571 & 10,666 & 183,721 & 0,730 & 0,845 & 1,576 \\
\hline 9 & 11,880 & 164,312 & 0,646 & 0,910 & 1,556 & 10,159 & 192,738 & 0,777 & 0,814 & 1,591 \\
\hline 10 & 11,846 & 168,800 & 0,627 & 0,937 & 1,564 & 10,530 & 192,482 & 0,734 & 0,836 & 1,569 \\
\hline 11 & 11,592 & 172,596 & 0,656 & 0,913 & 1,569 & 10,338 & 190,327 & 0,747 & 0,836 & 1,583 \\
\hline 12 & 11,395 & 169,657 & 0,657 & 0,905 & 1,562 & 10,191 & 199,538 & 0,806 & 0,793 & 1,598 \\
\hline 13 & 11,510 & 172,852 & 0,677 & 0,895 & 1,571 & 10,523 & 182,148 & 0,726 & 0,853 & 1,578 \\
\hline 14 & 11,411 & 177,093 & 0,678 & 0,887 & 1,565 & 10,104 & 198,092 & 0,788 & 0,801 & 1,589 \\
\hline 15 & 11,613 & 172,275 & 0,645 & 0,902 & 1,547 & 10,324 & 190,973 & 0,770 & 0,814 & 1,584 \\
\hline 16 & 13,071 & 162,012 & 0,546 & 0,968 & 1,515 & 10,330 & 191,481 & 0,774 & 0,815 & 1,589 \\
\hline 17 & 10,910 & 186,706 & 0,685 & 0,860 & 1,545 & 10,026 & 194,188 & 0,790 & 0,816 & 1,606 \\
\hline 18 & 11,958 & 174,743 & 0,644 & 0,897 & 1,541 & 10,433 & 194,702 & 0,761 & 0,803 & 1,563 \\
\hline 19 & 11,282 & 176,548 & 0,651 & 0,904 & 1,555 & 10,356 & 195,886 & 0,752 & 0,809 & 1,562 \\
\hline 20 & 11,552 & 173,027 & 0,683 & 0,900 & 1,583 & 10,355 & 195,335 & 0,775 & 0,818 & 1,594 \\
\hline 21 & 11,965 & 163,942 & 0,646 & 0,948 & 1,594 & 9,979 & 197,911 & 0,793 & 0,800 & 1,593 \\
\hline 22 & 12,024 & 168,963 & 0,638 & 0,921 & 1,560 & 10,742 & 192,097 & 0,749 & 0,828 & 1,576 \\
\hline 23 & 12,369 & 168,613 & 0,605 & 0,930 & 1,535 & 10,294 & 190,181 & 0,755 & 0,815 & 1,570 \\
\hline 24 & 11,178 & 180,799 & 0,672 & 0,885 & 1,557 & 10,081 & 201,823 & 0,790 & 0,775 & 1,564 \\
\hline 25 & 11,996 & 172,929 & 0,638 & 0,927 & 1,565 & 10,700 & 190,307 & 0,770 & 0,805 & 1,575 \\
\hline
\end{tabular}


WILEY-VCH

Table 2S3: Data of SLN at $10 \mathrm{mN}$.

\begin{tabular}{|c|c|c|c|c|c|c|c|c|c|c|}
\hline Polarization & Upward & Upward & Upward & Upward & Upward & Downward & Downward & Downward & Downward & Downward \\
\hline Property & Hardness & $\begin{array}{l}\text { Contact } \\
\text { Stiffness }\end{array}$ & $\begin{array}{l}\text { Plastic } \\
\text { energy }\end{array}$ & $\begin{array}{l}\text { Elastic } \\
\text { energy }\end{array}$ & $\begin{array}{c}\text { Total } \\
\text { energy }\end{array}$ & Hardness & $\begin{array}{l}\text { Contact } \\
\text { Stiffness }\end{array}$ & $\begin{array}{l}\text { Plastic } \\
\text { energy }\end{array}$ & $\begin{array}{l}\text { Elastic } \\
\text { energy }\end{array}$ & $\begin{array}{c}\text { Total } \\
\text { energy }\end{array}$ \\
\hline Units & GPa & $\mathrm{mN} / \mu \mathrm{m}$ & $\mathbf{n J}$ & $\mathbf{n J}$ & $\mathbf{n J}$ & GPa & $\mathrm{mN} / \mu \mathrm{m}$ & $\mathbf{n J}$ & $\mathbf{n J}$ & $\mathbf{n J}$ \\
\hline 1 & 12,431 & 145,385 & 0,346 & 0,470 & 0,816 & 11,921 & 127,538 & 0,222 & 0,567 & 0,789 \\
\hline 2 & 11,478 & 148,279 & 0,379 & 0,467 & 0,845 & 11,791 & 160,557 & 0,360 & 0,424 & 0,784 \\
\hline 3 & 11,933 & 139,480 & 0,359 & 0,490 & 0,849 & 11,017 & 160,989 & 0,385 & 0,432 & 0,817 \\
\hline 4 & 11,922 & 143,935 & 0,346 & 0,484 & 0,830 & 10,548 & 160,362 & 0,434 & 0,425 & 0,859 \\
\hline 5 & 12,071 & 144,321 & 0,361 & 0,469 & 0,830 & 10,907 & 154,533 & 0,390 & 0,443 & 0,833 \\
\hline 6 & 11,877 & 147,014 & 0,343 & 0,472 & 0,815 & 11,378 & 147,944 & 0,377 & 0,452 & 0,829 \\
\hline 7 & 13,428 & 136,607 & 0,315 & 0,491 & 0,806 & 10,790 & 147,055 & 0,392 & 0,454 & 0,846 \\
\hline 8 & 13,348 & 136,957 & 0,302 & 0,494 & 0,796 & 10,842 & 154,114 & 0,388 & 0,445 & 0,834 \\
\hline 9 & 12,589 & 141,085 & 0,339 & 0,477 & 0,816 & 11,036 & 150,941 & 0,393 & 0,462 & 0,856 \\
\hline 10 & 11,953 & 146,644 & 0,349 & 0,475 & 0,825 & 10,456 & 158,281 & 0,412 & 0,441 & 0,853 \\
\hline 11 & 12,740 & 143,148 & 0,325 & 0,475 & 0,801 & 10,986 & 155,164 & 0,383 & 0,453 & 0,837 \\
\hline 12 & 11,900 & 145,102 & 0,360 & 0,474 & 0,834 & 10,454 & 161,384 & 0,421 & 0,426 & 0,848 \\
\hline 13 & 12,219 & 144,097 & 0,330 & 0,476 & 0,806 & 11,985 & 141,094 & 0,357 & 0,461 & 0,819 \\
\hline 14 & 12,490 & 141,871 & 0,329 & 0,483 & 0,812 & 11,337 & 151,634 & 0,380 & 0,450 & 0,830 \\
\hline 15 & 12,419 & 145,231 & 0,334 & 0,480 & 0,814 & 10,163 & 162,240 & 0,415 & 0,437 & 0,852 \\
\hline 16 & 14,243 & 137,737 & 0,270 & 0,513 & 0,783 & 10,452 & 155,013 & 0,400 & 0,445 & 0,845 \\
\hline 17 & 12,333 & 138,936 & 0,354 & 0,484 & 0,838 & 11,412 & 150,910 & 0,375 & 0,455 & 0,831 \\
\hline 18 & 12,669 & 143,893 & 0,338 & 0,465 & 0,803 & 10,748 & 154,230 & 0,400 & 0,445 & 0,845 \\
\hline 19 & 12,884 & 142,870 & 0,322 & 0,487 & 0,809 & 10,935 & 155,900 & 0,399 & 0,452 & 0,851 \\
\hline 20 & 11,807 & 148,930 & 0,355 & 0,466 & 0,821 & 11,380 & 150,086 & 0,374 & 0,455 & 0,829 \\
\hline 21 & 12,816 & 144,874 & 0,318 & 0,474 & 0,791 & 10,486 & 165,828 & 0,407 & 0,429 & 0,836 \\
\hline 22 & 12,023 & 150,711 & 0,358 & 0,461 & 0,819 & 10,580 & 148,512 & 0,377 & 0,461 & 0,838 \\
\hline 23 & 12,269 & 145,013 & 0,343 & 0,477 & 0,820 & 11,101 & 152,827 & 0,390 & 0,459 & 0,848 \\
\hline 24 & 12,056 & 150,027 & 0,341 & 0,458 & 0,799 & 11,118 & 148,367 & 0,397 & 0,460 & 0,857 \\
\hline 25 & 12,738 & 145,160 & 0,321 & 0,473 & 0,794 & 10,444 & 165,368 & 0,417 & 0,426 & 0,842 \\
\hline
\end{tabular}

Table 2S4: Data of SLN at $7 \mathrm{mN}$.

\begin{tabular}{|c|c|c|c|c|c|c|c|c|c|c|}
\hline Polarization & Upward & Upward & Upward & Upward & Upward & Downward & Downward & Downward & Downward & Downward \\
\hline Property & Hardness & $\begin{array}{l}\text { Contact } \\
\text { Stiffness }\end{array}$ & $\begin{array}{l}\text { Plastic } \\
\text { energy }\end{array}$ & $\begin{array}{l}\text { Elastic } \\
\text { energy }\end{array}$ & $\begin{array}{l}\text { Total } \\
\text { energy }\end{array}$ & Hardness & $\begin{array}{l}\text { Contact } \\
\text { Stiffness }\end{array}$ & $\begin{array}{l}\text { Plastic } \\
\text { energy }\end{array}$ & $\begin{array}{l}\text { Elastic } \\
\text { energy }\end{array}$ & $\begin{array}{c}\text { Total } \\
\text { energy }\end{array}$ \\
\hline Units & GPa & $\mathrm{mN} / \mu \mathrm{m}$ & $\mathbf{n J}$ & $\mathbf{n J}$ & $\mathbf{n J}$ & GPa & $\mathrm{mN} / \mu \mathrm{m}$ & $\mathbf{n J}$ & $\mathbf{n J}$ & nJ \\
\hline 1 & 12,735 & 126,621 & 0,197 & 0,267 & 0,463 & 12,901 & 130,945 & 0,222 & 0,248 & 0,470 \\
\hline 2 & 13,278 & 118,582 & 0,182 & 0,279 & 0,461 & 11,911 & 118,717 & 0,205 & 0,277 & 0,483 \\
\hline 3 & 12,655 & 119,013 & 0,196 & 0,276 & 0,472 & 11,062 & 130,169 & 0,220 & 0,260 & 0,479 \\
\hline 4 & 12,645 & 120,772 & 0,188 & 0,280 & 0,468 & 11,941 & 117,123 & 0,205 & 0,282 & 0,486 \\
\hline 5 & 13,368 & 120,187 & 0,185 & 0,274 & 0,460 & 11,652 & 129,079 & 0,203 & 0,266 & 0,469 \\
\hline 6 & 12,283 & 125,358 & 0,202 & 0,270 & 0,472 & 12,147 & 125,128 & 0,200 & 0,272 & 0,472 \\
\hline 7 & 12,847 & 119,062 & 0,187 & 0,277 & 0,464 & 8,719 & 118,718 & 0,209 & 0,280 & 0,489 \\
\hline 8 & 13,477 & 113,793 & 0,188 & 0,280 & 0,467 & 11,392 & 132,841 & 0,215 & 0,258 & 0,473 \\
\hline 9 & 12,179 & 125,287 & 0,205 & 0,260 & 0,465 & 11,616 & 126,065 & 0,205 & 0,268 & 0,473 \\
\hline 10 & 13,304 & 115,695 & 0,181 & 0,285 & 0,467 & 11,888 & 124,038 & 0,208 & 0,268 & 0,476 \\
\hline 11 & 12,303 & 120,601 & 0,204 & 0,269 & 0,474 & 10,987 & 128,637 & 0,238 & 0,257 & 0,495 \\
\hline 12 & 13,937 & 113,795 & 0,159 & 0,295 & 0,455 & 12,569 & 121,063 & 0,194 & 0,274 & 0,468 \\
\hline 13 & 12,831 & 117,681 & 0,195 & 0,282 & 0,477 & 11,638 & 127,355 & 0,208 & 0,264 & 0,472 \\
\hline 14 & 13,755 & 119,058 & 0,179 & 0,277 & 0,456 & 12,149 & 126,621 & 0,198 & 0,265 & 0,463 \\
\hline 15 & 12,960 & 122,723 & 0,193 & 0,268 & 0,461 & 11,906 & 129,819 & 0,213 & 0,260 & 0,473 \\
\hline 16 & 13,631 & 123,402 & 0,170 & 0,276 & 0,446 & 11,966 & 125,447 & 0,198 & 0,271 & 0,470 \\
\hline 17 & 13,897 & 119,838 & 0,169 & 0,277 & 0,446 & 11,314 & 125,828 & 0,212 & 0,269 & 0,481 \\
\hline 18 & 12,866 & 117,559 & 0,199 & 0,281 & 0,479 & 12,012 & 117,272 & 0,205 & 0,278 & 0,483 \\
\hline 19 & 13,808 & 114,979 & 0,183 & 0,285 & 0,468 & 11,779 & 128,184 & 0,211 & 0,263 & 0,474 \\
\hline 20 & 12,889 & 123,703 & 0,190 & 0,268 & 0,458 & 12,266 & 120,868 & 0,203 & 0,273 & 0,476 \\
\hline 21 & 14,203 & 110,906 & 0,158 & 0,299 & 0,457 & 11,635 & 127,951 & 0,205 & 0,269 & 0,473 \\
\hline 22 & 13,886 & 118,674 & 0,170 & 0,288 & 0,457 & 11,983 & 128,037 & 0,203 & 0,263 & 0,466 \\
\hline 23 & 13,235 & 118,618 & 0,182 & 0,277 & 0,459 & 12,190 & 126,422 & 0,208 & 0,264 & 0,471 \\
\hline 24 & 12,425 & 122,340 & 0,191 & 0,270 & 0,461 & 12,300 & 140,134 & 0,225 & 0,246 & 0,471 \\
\hline 25 & 13,273 & 117,248 & 0,172 & 0,287 & 0,459 & 10,971 & 133,556 & 0,227 & 0,250 & 0,477 \\
\hline
\end{tabular}




\section{WILEY-VCH}

Table 2S5: Data of PPLN at $7 \mathrm{mN}$.

\begin{tabular}{|c|c|c|c|c|c|c|c|c|c|c|}
\hline Polarization & Upward & Upward & Upward & Upward & Upward & Downward & Downwar & Downward & Downward & Downward \\
\hline Property & Hardness & $\begin{array}{l}\text { Contact } \\
\text { Stiffness }\end{array}$ & $\begin{array}{l}\text { Plastic } \\
\text { energy }\end{array}$ & $\begin{array}{l}\text { Elastic } \\
\text { energy }\end{array}$ & $\begin{array}{c}\text { Total } \\
\text { energy }\end{array}$ & Hardness & $\begin{array}{l}\text { Contact } \\
\text { Stiffness }\end{array}$ & $\begin{array}{l}\text { Plastic } \\
\text { energy }\end{array}$ & $\begin{array}{l}\text { Elastic } \\
\text { energy }\end{array}$ & $\begin{array}{c}\text { Total } \\
\text { energy }\end{array}$ \\
\hline Units & GPa & $\mathrm{mN} / \mu \mathrm{m}$ & $\mathbf{n J}$ & nJ & $\mathbf{n J}$ & GPa & $\mathrm{mN} / \mu \mathrm{m}$ & $\mathbf{n J}$ & nJ & $\mathbf{n J}$ \\
\hline 1 & 12,701 & 142,265 & 0,231 & 0,246 & 0,477 & 12,049 & 149,684 & 0,243 & 0,231 & 0,474 \\
\hline 2 & 12,540 & 138,653 & 0,232 & 0,251 & 0,483 & 11,915 & 152,115 & 0,246 & 0,233 & 0,479 \\
\hline 3 & 13,226 & 137,928 & 0,220 & 0,247 & 0,467 & 13,231 & 143,113 & 0,213 & 0,251 & 0,464 \\
\hline 4 & 12,796 & 136,577 & 0,232 & 0,251 & 0,482 & 11,814 & 147,557 & 0,250 & 0,233 & 0,483 \\
\hline 5 & 12,327 & 138,788 & 0,243 & 0,237 & 0,480 & 12,361 & 145,708 & 0,233 & 0,239 & 0,472 \\
\hline 6 & 12,783 & 140,067 & 0,227 & 0,244 & 0,471 & 12,108 & 148,244 & 0,250 & 0,230 & 0,480 \\
\hline 7 & 12,855 & 141,007 & 0,230 & 0,241 & 0,471 & 11,914 & 151,948 & 0,238 & 0,233 & 0,471 \\
\hline 8 & 12,636 & 142,657 & 0,228 & 0,248 & 0,476 & 12,607 & 145,239 & 0,219 & 0,246 & 0,465 \\
\hline 9 & 12,337 & 142,962 & 0,229 & 0,243 & 0,473 & 12,772 & 140,802 & 0,220 & 0,246 & 0,467 \\
\hline 10 & 12,746 & 135,864 & 0,223 & 0,253 & 0,477 & 11,371 & 146,979 & 0,270 & 0,227 & 0,497 \\
\hline 11 & 12,665 & 143,138 & 0,240 & 0,242 & 0,482 & 13,977 & 132,083 & 0,206 & 0,259 & 0,465 \\
\hline 12 & 13,765 & 132,719 & 0,206 & 0,258 & 0,464 & 11,990 & 152,816 & 0,237 & 0,229 & 0,466 \\
\hline 13 & 12,413 & 140,034 & 0,230 & 0,247 & 0,477 & 11,856 & 147,911 & 0,246 & 0,234 & 0,480 \\
\hline 14 & 12,577 & 144,348 & 0,236 & 0,240 & 0,476 & 13,265 & 131,913 & 0,210 & 0,253 & 0,463 \\
\hline 15 & 12,438 & 141,000 & 0,233 & 0,246 & 0,479 & 11,801 & 146,527 & 0,255 & 0,228 & 0,483 \\
\hline 16 & 12,492 & 143,903 & 0,233 & 0,238 & 0,471 & 12,412 & 141,784 & 0,233 & 0,247 & 0,480 \\
\hline 17 & 12,665 & 137,454 & 0,227 & 0,249 & 0,477 & 13,639 & 139,530 & 0,208 & 0,249 & 0,458 \\
\hline 18 & 12,361 & 138,502 & 0,235 & 0,244 & 0,480 & 12,778 & 139,956 & 0,230 & 0,239 & 0,469 \\
\hline 19 & 13,539 & 135,379 & 0,212 & 0,258 & 0,469 & 12,598 & 138,116 & 0,229 & 0,248 & 0,477 \\
\hline 20 & 12,204 & 147,404 & 0,235 & 0,246 & 0,480 & 12,500 & 138,512 & 0,231 & 0,249 & 0,480 \\
\hline 21 & 12,311 & 142,288 & 0,232 & 0,245 & 0,476 & 12,865 & 136,117 & 0,229 & 0,252 & 0,481 \\
\hline 22 & 12,556 & 141,590 & 0,231 & 0,243 & 0,473 & & & & & \\
\hline 23 & 12,451 & 141,633 & 0,233 & 0,244 & 0,477 & & & & & \\
\hline 24 & 12,683 & 132,916 & 0,235 & 0,259 & 0,494 & & & & & \\
\hline 25 & 13,858 & 128,829 & 0,202 & 0,268 & 0,470 & & & & & \\
\hline 26 & 13,460 & 131,222 & 0,215 & 0,258 & 0,472 & & & & & \\
\hline 27 & 12,459 & 137,953 & 0,230 & 0,250 & 0,480 & & & & & \\
\hline 28 & 13,214 & 133,501 & 0,220 & 0,258 & 0,477 & & & & & \\
\hline 29 & 12,144 & 159,805 & 0,236 & 0,232 & 0,468 & & & & & \\
\hline 30 & 12,563 & 135,515 & 0,228 & 0,253 & 0,481 & & & & & \\
\hline 31 & 12,459 & 138,624 & 0,232 & 0,252 & 0,484 & & & & & \\
\hline 32 & 11,911 & 143,691 & 0,240 & 0,243 & 0,482 & & & & & \\
\hline 33 & 11,995 & 138,199 & 0,256 & 0,239 & 0,496 & & & & & \\
\hline 34 & 12,315 & 140,584 & 0,232 & 0,246 & 0,478 & & & & & \\
\hline 35 & 12,654 & 142,329 & 0,231 & 0,239 & 0,469 & & & & & \\
\hline 36 & 13,778 & 130,052 & 0,210 & 0,266 & 0,476 & & & & & \\
\hline 37 & 13,223 & 129,109 & 0,219 & 0,263 & 0,482 & & & & & \\
\hline 38 & 12,885 & 135,440 & 0,221 & 0,253 & 0,473 & & & & & \\
\hline 39 & 12,011 & 138,366 & 0,241 & 0,247 & 0,488 & & & & & \\
\hline 40 & 13,801 & 133,601 & 0,207 & 0,254 & 0,461 & & & & & \\
\hline 41 & 11,738 & 146,899 & 0,244 & 0,239 & 0,483 & & & & & \\
\hline 42 & 13,062 & 136,646 & 0,219 & 0,250 & 0,468 & & & & & \\
\hline
\end{tabular}

\section{References}

[1] Zhou, H., Pei, Y., Li, F., Luo, H. \& Fang, D. Appl. Phys. Lett 2014, 104, 061904.

[2] Abdollahi, A., Peco, C., Millán, D., Arroyo, M., Catalán, G. \& Arias, I. Phys. Rev B 2015, 92, 094101.

[3] Devonshire, A.F. Phil. Mag 1949, 40, 1040.

[4] Zubko, P., Catalán, G. \& Tagantsev, A.K. Annu. Rev. Mater. Res. 2013, 43: 387 - 421.

[5] Sharma, N. D. , Landis C. M., and Sharma P., J. Appl. Phys. 2010, 108: 024304.

[6] Shu L., Wei X., Pang T., Yao X. and Wang C. J Appl Phys 2011, 110, 104106.

[7] Johnson K. L. J Mech Phys Solids 1970, 18, 115-126. 
WILEY-VCH 